\title{
Interface effects in the magneto-optical properties of Co nanoparticles in dielectric matrix
}

\author{
C. Clavero ${ }^{\text {a) }}$ and G. Armelles \\ Instituto de Microelectrónica de Madrid-IMM (CNM-CSIC), Isaac Newton 8-PTM, E-28760, Tres Cantos, \\ Madrid, Spain \\ J. Margueritat and J. Gonzalo \\ Laser Processing Group, Instituto de Óptica, CSIC, Serrano 121, E-28006 Madrid, Spain \\ M. García del Muro, A. Labarta, and X. Batlle \\ Department de Física Fonamental, Universitat de Barcelona, Martí i Franquès 1, E-08028 Barcelona, \\ Spain and Institut de Nanociència i Nanotecnologia, Universitat de Barcelona, Martí i Franquès 1, \\ E-08028 Barcelona, Spain
}

(Received 7 March 2007; accepted 11 April 2007; published online 4 May 2007)

\begin{abstract}
The authors present a study of the optical and magneto-optical properties of Co nanoparticles embedded in two amorphous dielectric matrices with different refractive indices such as $\mathrm{ZrO}_{2}$ and $\mathrm{Al}_{2} \mathrm{O}_{3}$. The nanostructured films were prepared by pulsed laser deposition, and the morphology and structure were studied by different characterization techniques. The optical and magneto-optical (MO) properties of the Co inside the nanoparticles differ from those of the bulk material; in particular, a decrease in the MO constants is found. These properties are found to depend on the nanoparticle size and on the dielectric matrix, due to the different nanoparticle-matrix interfaces appearing in both cases. (C) 2007 American Institute of Physics. [DOI: 10.1063/1.2737126]
\end{abstract}

Materials consisting of nanoparticles embedded in dielectric matrices are attracting a great interest due to their physical properties, which differ considerably from the bulk properties of the constituent materials. These modified properties arise from different aspects, such as size and surface effects. ${ }^{1}$ In particular, the large fraction of atoms located at the surface makes the nanoparticles very sensitive to the medium surrounding them, giving rise to strong changes in their properties. To name a few, modifications in the magnetic anisotropy have been found in nanoparticles due to surface effects depending on the embedding matrix, ${ }^{2}$ and the onset of permanent magnetism in thiol-capped Au nanoparticles has been described. ${ }^{3}$ Regarding the optical response in systems consisting of embedded nanoparticles, surface effects have been found in $\mathrm{Au}$ and $\mathrm{Ag}$ nanoparticles on a variety of matrices $^{4,5}$ that are attributed to modifications of the electron density profile normal to the nanoparticle surface, which affect the scattering mechanism of the latter. Nevertheless, these surface effects have not been previously described in the magneto-optical (MO) behavior of magnetic nanoparticles.

For this study, two systems consisting of Co nanoparticles embedded in two different dielectric matrices such as $\mathrm{ZrO}_{2}(n \sim 2$ in the considered spectral range $1.4-4.3 \mathrm{eV})$ and $\mathrm{Al}_{2} \mathrm{O}_{3}(n \sim 1.76)$ were prepared. A $200 \mathrm{~nm}$ thick $\mathrm{Co}-\mathrm{ZrO}_{2}$ granular film was grown on $\mathrm{Si}$ substrates in a vacuum chamber $\left(p=10^{-4}\right.$ mbar) equipped with a $\mathrm{KrF}$ pulsed laser deposition (PLD) system at room temperature, leading to a Co volume concentration of $20 \%$ in the film as deduced by an electron probe microanalyzer Cameca SX-50. ${ }^{6}$ In the second case, a $\mathrm{Co}-\mathrm{Al}_{2} \mathrm{O}_{3}$ multilayer system was prepared in high vacuum $\left(p<10\right.$ mbar) by alternated $\mathrm{PLD}$ on a $\mathrm{SiO}_{2}$ substrate. The structure of the samples is described elsewhere, ${ }^{7}$

\footnotetext{
a) Author to whom correspondence should be addressed; electronic mail:
} cesarcl@imm.cnm.csic.es with a Co concentration of $25 \%$ in each $\mathrm{Co}-\mathrm{Al}_{2} \mathrm{O}_{3}$ film being found, as deduced by Rutherford backscattering (RBS).

The morphology and structure of the systems were studied by means of high resolution transmission electron microscopy, x-ray diffraction, x-ray reflectrometry, RBS, and microprobe. More information about the structural and morphological characterization of both systems can be found elsewhere. ${ }^{6,7}$ In the case of the $\mathrm{Co}-\mathrm{ZrO}_{2}$ system, the $\mathrm{Co}$ nanoparticles exhibit sharp Co-matrix interfaces, with a mean nanoparticle diameter of $D=2.5 \mathrm{~nm}$ and a $20 \mathrm{vol} \%$ Co concentration in the layer. On the other hand, in the Co- $\mathrm{Al}_{2} \mathrm{O}_{3}$ system, a mean nanoparticle diameter of $D$ $=2 \mathrm{~nm}$ was measured, with a $25 \mathrm{vol} \%$ Co concentration in each $3 \mathrm{~nm}$ thick $\mathrm{Co}-\mathrm{Al}_{2} \mathrm{O}_{3}$ layer of the multilayer structure. In this case, no clear information on the Co-matrix interface could be extracted from the structural characterization. Previous magnetic characterization of these samples ${ }^{7}$ discounts the formation of a magnetic $\mathrm{CoO}$ shell, although the formation of other oxides, as in the case of Fe nanoparticles produced in the same experimental conditions, ${ }^{8}$ cannot be ruled out.

The optical constants corresponding to the $\mathrm{Co}-\mathrm{ZrO}_{2}$ and $\mathrm{Co}-\mathrm{Al}_{2} \mathrm{O}_{3}$ films measured by ellipsometry in the $1.4-4.2 \mathrm{eV}$ spectral range are depicted in Fig. 1, together with the optical constants of a polycrystalline thick continuous Co film for comparison. The values of the refractive index $(n)$ of both Co nanoparticle systems are very similar. On the other hand, the absorption coefficient $(k)$ shows a broad band. The intensity of the band and the energy position of the maximum depend on the matrix: the intensity increases and the position of the maximum shifts to lower energies as we increase the refractive index of the matrix. This dependence with the optical properties of the matrix is the expected behavior for a localized surface plasmon resonance (LSPR) of a metallic nanoparticles system. ${ }^{5}$ Therefore, we attribute the broad band observed in the absorption coefficient to a LSPR of the Co 


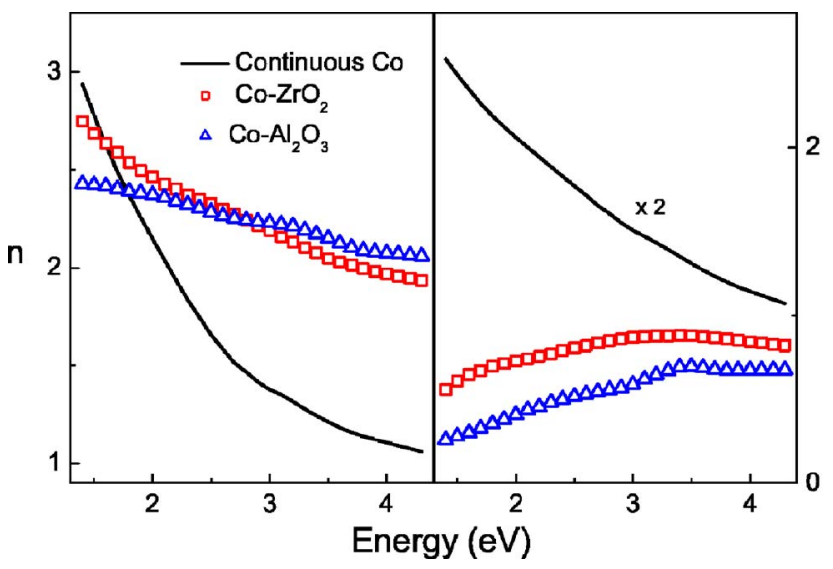

FIG. 1. (Color online) Optical constants ( $n$ and $k$ ) for the $\mathrm{Co}-\mathrm{ZrO}_{2}$ and $\mathrm{Co}-\mathrm{Al}_{2} \mathrm{O}_{3}$ films measured by ellipsometry. For comparison, the optical constants of a polycrystalline thick continuous Co film are also shown.

nanoparticles. Additionally, the MO constants $\left(\varepsilon_{x y}\right)$ of the films (Fig. 2) were derived from the measured polar Kerr rotation and ellipticity spectra, taking into account the experimental optical constants presented in Fig. 1. The $\mathrm{Co}-\mathrm{ZrO}_{2}$ film exhibits higher MO constants in all the spectral range, crossing the real part the energy axis at a lower energy values.

Due to the size of the nanoparticles, the optical and MO properties of granular systems can be described by using an effective medium approximation, ${ }^{9,10}$ and for the present nanoparticle concentration, the most suitable approximation is the so-called Maxwell-Garnett effective medium model. ${ }^{11}$ From the measured optical and MO constants of the films (Figs. 1 and 2), assuming that the nanoparticles have spherical shape as observed by transmission electron microscopy ${ }^{6,7}$ and considering the measured volume Co concentration, we can obtain the optical [Fig 3(a)] and the MO constants [Fig $3(\mathrm{~b})]$ of the nanoparticles' Co. The value of $n$ corresponding to the Co extracted from the $\mathrm{Co}-\mathrm{Al}_{2} \mathrm{O}_{3}$ film exceeds the $\mathrm{Co}$ polycrystalline value in all the considered spectral range, whereas the value of $n$ corresponding to the Co extracted from the $\mathrm{Co}-\mathrm{ZrO}_{2}$ film is very similar to it. On the other hand, the values obtained for $k$ in both cases are very similar and lower than the polycrystalline Co value. Regarding the MO constants, Fig. 3(b) shows a noticeable decrease in the real and imaginary parts with respect to the polycrystalline Co values in both systems, with small differences between the MO constants of the Co inside both films being found.

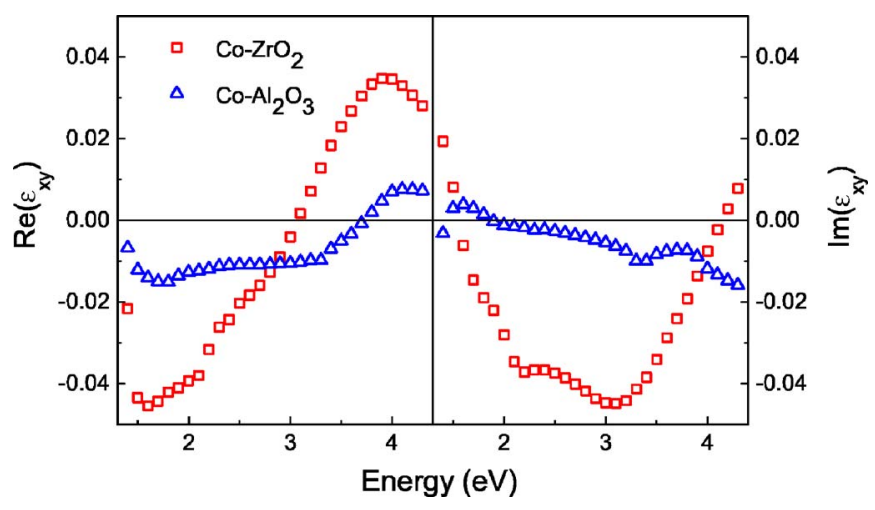

FIG. 2. (Color online) Real and imaginary parts of the magneto-optical

constants $\left(\varepsilon_{x y}\right)$ for the $\mathrm{Co}-\mathrm{ZrO}_{2}$ and $\mathrm{Co}-\mathrm{Al}_{2} \mathrm{O}_{3}$ films. Drude model ${ }^{15}$ as follows: ${ }^{16}$
Downloaded 09 Mar 2010 to 161.111.180.191. Redistribution subject to AlP license or copyright; see http://apl.aip.org/apl/copyright.jsp (a)

(b)
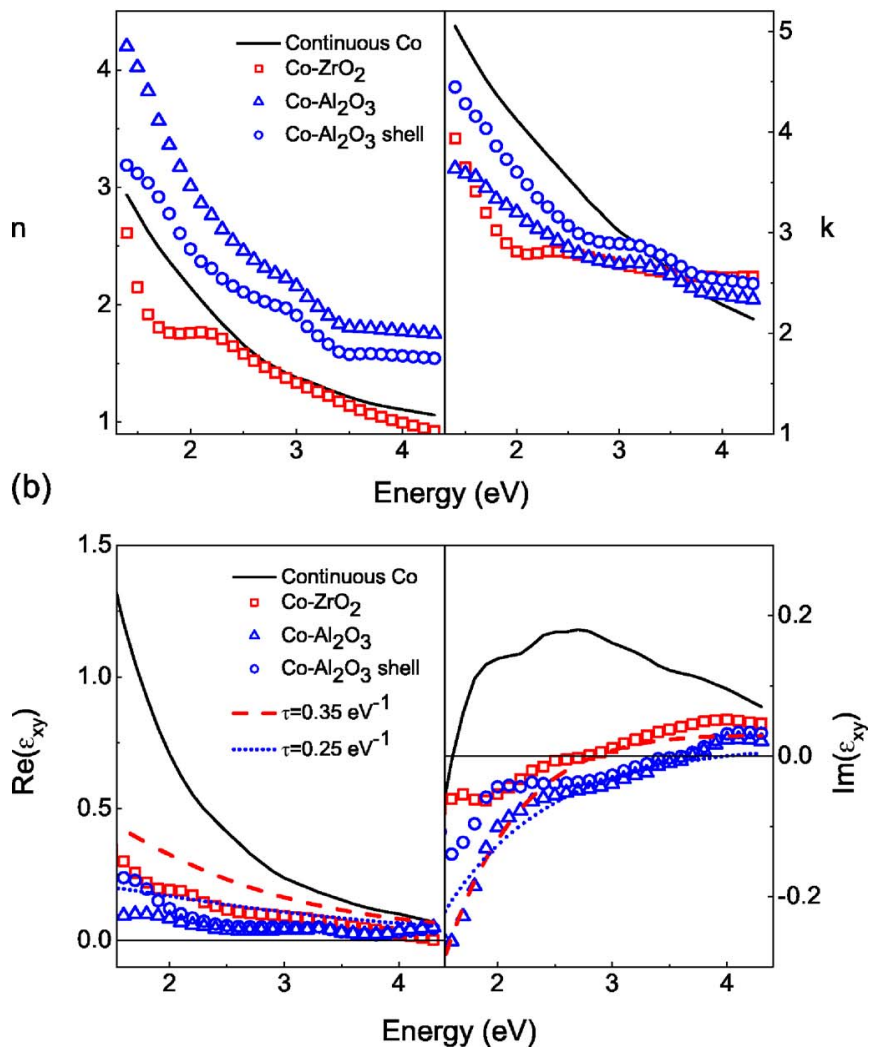

FIG. 3. (Color online) (a) Optical and (b) magneto-optical constants of the $\mathrm{Co}$ being part of the nanoparticles embedded in $\mathrm{ZrO}_{2}$ and $\mathrm{Al}_{2} \mathrm{O}_{3}$. For comparison, the constants of a polycrystalline thick continuous Co film are also shown. The constants calculated for the Co core in the $\mathrm{Co}-\mathrm{Al}_{2} \mathrm{O}_{3}$ system are represented by rounded symbols in (a) and (b). The dashed and dotted lines in (b) correspond to the intraband contribution obtained using the Drude model and with $\tau=035$ and $0.25 \mathrm{eV}^{-1}$, respectively.

As nanoparticle sizes are very similar in both matrices, the observed difference between the optical and MO constants of Co should be related to interface effects. A simple way to model these effects is to describe the nanoparticles as a complex system consisting of a core surrounded by a shell. ${ }^{12}$ For example, let us focus on the $\mathrm{Co}-\mathrm{Al}_{2} \mathrm{O}_{3}$ system, in which the Co-matrix interface was not clearly resolved. We suppose that the nanoparticles have a $1.5 \AA$ thick oxide shell and assume for this oxide layer the values of the optical constants of $\mathrm{Co}_{3} \mathrm{O}_{4}$ (Ref. 13) since we can discard the presence of $\mathrm{CoO}{ }^{7}$ By considering this assumption, we obtain the optical and MO constants of the Co core [referred as $\mathrm{Co}-\mathrm{Al}_{2} \mathrm{O}_{3}$ shell in Fig. 3(a) and 3(b)], which are very similar to those found for the Co inside the $\mathrm{Co}-\mathrm{ZrO}_{2}$. This finding supports the idea that the differences observed in the optical and MO constants of Co inside the two matrices are due to interface effects.

Going back to the differences observed between the MO constants of these two systems and those of the bulk material, they should be related to size effects. The dielectric tensor of metallic systems has two predominant contributions: intraband (owing to conduction electrons in the material) and interband (due to interband transitions) contributions. In the case of $\mathrm{Co}$, it has been shown in previous works ${ }^{14,17}$ that the MO properties can be described very accurately by only considering the intraband contribution, owing to conduction electrons, by means of a free electron Drude model ${ }^{15}$ as follows: ${ }^{16}$ 


$$
\varepsilon_{x y}^{\text {intraband }}(\omega)=\frac{\omega_{c}\left(\omega_{p} \tau\right)^{2}}{\omega\left[(1-i \omega \tau)^{2}+\left(\omega_{c} \tau\right)^{2}\right]},
$$

where $\omega_{p}=\sqrt{4 \pi n e^{2} / m}$ is the plasma frequency, $\omega_{c}=e B / m c$ is the cyclotron frequency, and $\tau$ is the relaxation time of the electrons, which depends on the electron-electron, electronphonon, and electron-defect scattering contributions. In granular layers, such as those studied here, the relaxation time of the electrons, related to the electron mean free path, ${ }^{5}$ is considerably reduced due to the breaking of the lattice periodicity and the collisions of the electrons at the nanoparticle surface. ${ }^{11,17}$ The dependence of the relaxation time of the electrons on the size of the nanoparticles can be expressed as ${ }^{5,18}$

$$
\frac{1}{\tau}=\frac{1}{\tau_{0}}+\frac{A}{R},
$$

where $R$ is the radius of the nanoparticle, $\tau_{0}$ is the relaxation time of the electrons in the bulk material, and $A$ is a constant that depends mainly on the material and on the shape of the nanoparticles. In previous works, from the analysis of the optical properties of $\mathrm{Au}$ and $\mathrm{Ag}$ nanoparticles, ${ }^{4,19}$ strong differences have been found in the dependence of the relaxation time of the electrons $(\tau)$ with the size of the nanoparticles as a function of the embedding matrix, giving rise to variations in the $A$ constant attributed to nanoparticle-matrix interface effects. ${ }^{4}$ In this letter, a Drude-like evolution has been assumed in the MO constants, taking the parameters obtained by Krinchik ${ }^{20,21}$ for polycrystalline $\mathrm{Co}\left(\omega_{p}=9.74 \mathrm{eV}, \omega_{c}\right.$ $=0.089 \mathrm{eV}$, and $\tau=0.632 \mathrm{eV}^{-1}=4.16 \times 10^{-16} \mathrm{~s}$ ) and varying only the relaxation time of the electrons $(\tau)$ to properly fit the experimental spectra [Fig. 3(b)]. In the case of the Co nanoparticles embedded in a $\mathrm{ZrO}_{2}$ matrix, a relaxation time of $\tau$ $=0.35 \mathrm{eV}^{-1}=2.3 \times 10^{-16} \mathrm{~s}$ was obtained in order to reproduce the MO response (dashed line), whereas a lower relaxation time of $\tau=0.25 \mathrm{eV}^{-1}=1.64 \times 10^{-16} \mathrm{~s}$ was obtained for the $\mathrm{Co}$ nanoparticles embedded in $\mathrm{Al}_{2} \mathrm{O}_{3}$ (dotted line). The differences found in the relaxation time of the electrons in both cases cannot be only attributed to the slightly different size of the nanoparticles $(R=1.25 \mathrm{~nm}$ in the nanoparticles embedded in $\mathrm{ZrO}_{2}$ and $R=1 \mathrm{~nm}$ in those embedded in $\mathrm{Al}_{2} \mathrm{O}_{3}$ ) but may also be due to modifications in the $A$ constant. By means of Eq. (2), a value of $A=1.6 \mathrm{~nm} \mathrm{eV}$ for the nanoparticles embedded in $\mathrm{ZrO}_{2}$ and a higher value of $A$ $=2.4 \mathrm{~nm} \mathrm{eV}$ for the nanoparticles embedded in $\mathrm{Al}_{2} \mathrm{O}_{3}$ were calculated. These differences found in the $A$ constant can be attributed to interface effects related to the different nanoparticle-matrix interfaces found in both systems (on one hand, sharp $\mathrm{Co}-\mathrm{ZrO}_{2}$ interfaces and, on the other hand, Co nanoparticles surrounded by a $\mathrm{Co}_{3} \mathrm{O}_{4}$ shell in the $\mathrm{Co}-\mathrm{Al}_{2} \mathrm{O}_{3}$ system), which cause differences in the electron density profile normal to the nanoparticle surface and therefore variations in the dependence of the relaxation time of the electrons $(\tau)$ with the nanoparticle size. A higher value of $A$ in the case of the Co nanoparticles embedded in $\mathrm{Al}_{2} \mathrm{O}_{3}$ leads to a stronger dependence of the relaxation time of the electrons with the size of the nanoparticles, which in this case is compatible with the proposed existence of an amorphous $\mathrm{Co}_{3} \mathrm{O}_{4}$ shell surrounding the Co core of the nanoparticles.

In conclusion, the optical and MO properties of two systems consisting of Co nanoparticles with similar concentration $(\sim 20 \%)$ and nanoparticle size $(D \sim 2 \mathrm{~nm})$ but embedded in two dielectric matrices with different refractive indices such as $\mathrm{ZrO}_{2}(n \sim 2)$ and $\mathrm{Al}_{2} \mathrm{O}_{3}(n \sim 1.76)$ have been studied. Using an effective medium approximation, the optical and MO properties of the Co nanoparticles are obtained, which differ from those of Co bulk material and depend on the size of the nanoparticles and on the matrix. It has been shown that the nanoparticle-matrix interface effects influence more strongly the optical constants, with the changes observed in the MO properties being smaller.

This work was financed by the Spanish CICYT (MAT2005-06508-C02-01 and MAT2006-03999) and Catalan DURSI (2005SGR00969). Two of the authors (C.C. and J.M.) acknowledge financial support from the MEC through FPI program and from the ESF through I3P program. The authors thank R. Serna (IO-CSIC) for help with the ellipsometric measurements and J. García López (CNA-CSIC) for RBS characterization.

\footnotetext{
${ }^{1}$ X. Batlle and A. Labarta, J. Phys. D 35, R15 (2002).

${ }^{2}$ F. Luis, F. Bartolomé, F. Petroff, J. Bartolomé, L. M. García, C. Deranlot, H. Jaffrès, M. J. Martínez, P. Bencok, F. Wilhelm, A. Rogalev, and N. B. Brookes, Europhys. Lett. 76, 142 (2006).

${ }^{3}$ P. Crespo, R. Litrán, T. C. Rojas, M. Multigner, J. M. de la Fuente, J. C. Sánchez-López, M. A. García, A. Hernando, S. Penadés, and A. Fernández, Phys. Rev. Lett. 93, 087204 (2004).

${ }^{4}$ U. Kreibig and L. Genzel, Surf. Sci. 156, 678 (1985).

${ }^{5}$ U. Kreibig and M. Vollmer, Optical Properties of Metal Clusters (Springer, Berlin, 1995), Vol. 25, p. 36.

${ }^{6}$ B. J. Hattink, M. García del Muro, Z. Konstantinović, X. Batlle, A. Labarta, and M. Varela, Phys. Rev. B 73, 045418 (2006).

${ }^{7}$ A. N. Dobrynin, D. N. Ievlev, K. Temst, P. Lievens, J. Margueritat, J. Gonzalo, C. N. Afonso, S. Q. Zhou, A. Vantomme, E. Piscopiello, and G. Van Tendeloo, Appl. Phys. Lett. 87, 012501 (2005).

${ }^{8}$ N. M. Dempsey, L. Ranno, D. Givord, J. Gonzalo, R. Serna, G. T. Fei, A. K. Petford-Long, R. C. Doole, and D. E. Hole, J. Appl. Phys. 90, 6268 (2001).

${ }^{9}$ D. Stroud, Phys. Rev. B 12, 3368 (1975).

${ }^{10}$ J. L. Menéndez, B. Bescós, G. Armelles, R. Serna, J. Gonzalo, R. Doole, A. K. Petford-Long, and M. I. Alonso, Phys. Rev. B 65, 205413 (2002).

${ }^{11}$ C. Clavero, Y. Huttel, A. Cebollada, G. Armelles, J. Arbiol, F. Peiró, and A. Cornet, Phys. Rev. B 72, 024441 (2005).

${ }^{12}$ M. Abe and T. Suwa, Phys. Rev. B 70, 235103 (2004).

${ }^{13}$ B. Rellinghaus, S. Fernandez de Avila, D. Weller, G. Armelles, R. Beyers, and A. Kellock, J. Appl. Phys. 83, 5621 (1998).

${ }^{14}$ C. Clavero, Y. Huttel, A. Cebollada, G. Armelles, J. Arbiol, F. Peiró, and A. Cornet, Phys. Rev. B 72, 024441 (2005).

${ }^{15}$ P. B. Johnson and R. W. Christy, Phys. Rev. B 6, 4370 (1972).

${ }^{16}$ T. K. Xia, P. M. Hui, and D. Stroud, J. Appl. Phys. 67, 2736 (1990).

${ }^{17}$ C. Clavero, B. Sepúlveda, G. Armelles, Z. Konstantinović, M. García del Muro, A. Labarta, and X. Batlle, J. Appl. Phys. 100, 074320 (2006).

${ }^{18}$ S. Norrman, T. Andersson, C. G. Granqvist, and O. Hunderi, Phys. Rev. B 18, 674 (1978).

${ }^{19}$ H. Hövel, S. Fritz, A. Hilger, U. Kreibig, and M. Vollmer, Phys. Rev. B 48, 18178 (1993).

${ }^{20}$ G. S. Krinchik, J. Appl. Phys. 35, 1089 (1964).

${ }^{21}$ R. Carey and B. W. J. Thomas, J. Phys. D 7, 2362 (1974).
} 